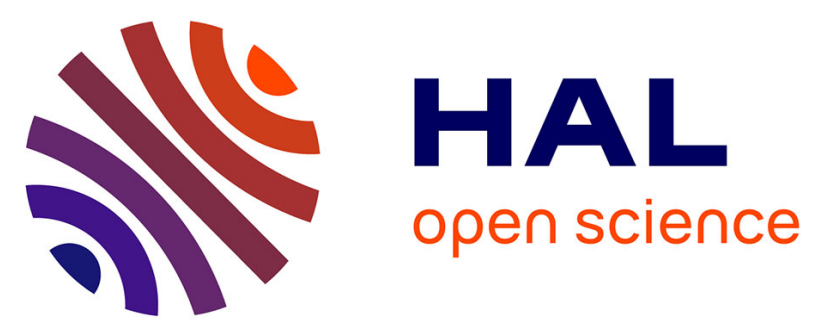

\title{
Effect of integrated palliative care on the quality of end-of-life care: retrospective analysis of 521 cancer patients
}

\author{
Isabelle Colombet, Vincent Montheil, Jean-Philippe Durand, Florence \\ Gillaizeau, Ralph Niarra, Cécile Jaeger, Jérôme Alexandre, François \\ Goldwasser, Pascale Vinant
}

\section{To cite this version:}

Isabelle Colombet, Vincent Montheil, Jean-Philippe Durand, Florence Gillaizeau, Ralph Niarra, et al.. Effect of integrated palliative care on the quality of end-of-life care: retrospective analysis of 521 cancer patients. BMJ Palliative and supportive care, 2012, 2 (3), pp.239-247. 10.1136/bmjspcare2011-000157 . hal-01295713

\section{HAL Id: hal-01295713 \\ https://hal-univ-paris.archives-ouvertes.fr/hal-01295713}

Submitted on 1 Apr 2016

HAL is a multi-disciplinary open access archive for the deposit and dissemination of scientific research documents, whether they are published or not. The documents may come from teaching and research institutions in France or abroad, or from public or private research centers.
L'archive ouverte pluridisciplinaire HAL, est destinée au dépôt et à la diffusion de documents scientifiques de niveau recherche, publiés ou non, émanant des établissements d'enseignement et de recherche français ou étrangers, des laboratoires publics ou privés.

\section{(ㅇ)(1) $\$$}

Distributed under a Creative Commons Attribution - NonCommercial - NoDerivatives $\mid 4.0$ 


\section{Effect of integrated palliative care on the quality of end of life care: retrospective analysis of 521 cancer patients.}

Isabelle Colombet, $\mathrm{MD}, \mathrm{PhD}^{\mathrm{a}, \mathrm{b}, \mathrm{c}}$

Vincent Montheil, MD ${ }^{\mathrm{c}}$

Jean-Philippe Durand, MD ${ }^{\mathrm{d}}$

Florence Gillaizeau, MSc ${ }^{\text {a,b }}$

Ralph Niarra, MSc ${ }^{\mathrm{b}}$

Cécile Jaeger, $\mathrm{MD}^{\mathrm{c}}$

Jérôme Alexandre, $\mathrm{MD}, \mathrm{PhD}^{\mathrm{a}, \mathrm{d}}$

François Goldwasser, $\mathrm{MD}, \mathrm{PhD}{ }^{\mathrm{a}, \mathrm{d}}$

Pascale Vinant, $\mathrm{MD}^{\mathrm{c}}$

aUniversité Paris Descartes, Sorbonne Paris Cité, Faculté de Médecine, LEM - EA 4569, Paris, France

${ }^{\mathrm{b}}$ INSERM, Centre d'Investigation Épidémiologique 4, Paris, France

${ }^{\mathrm{c}}$ PalliativeMedicine, Cochin Teaching Hospital, AP-HP, Paris, France

d, Medical oncology, Cochin Teaching Hospital, AP-HP, Paris, France

Corresponding author: Isabelle Colombet, Unité fonctionnelle de Médecine Palliative, Hôpital Cochin, 27, rue du Faubourg Saint-Jacques 75679 PARIS Cedex 14 ; e-mail : $\underline{\text { isabelle.colombet } @ \text { parisdescartes.fr }}$

Keywords: Palliative care; Oncology service, hospital ; Delivery of Health Care, Integrated; Health Care Quality, Access, and Evaluation; Retrospective study 
Word count: 3 595;

Number of tables : 4 ;

Number of figures : 2 


\section{ABSTRACT (267 words)}

Objective: To examine the impact, on three quality indicators of end-of-life (EOL) care, of oncologist's awareness in palliative care (PC), timing of the PC team (PCT) intervention, and multidisciplinary decision-making thanks to weekly onco-palliative meetings (OPMs).

Setting: France, Cochin academic hospital, 2007-2008

Design and participants: a 521 decedent case series study nested in a cohort of 735 metastatic cancer patients previously treated with chemotherapy.

Indicators : Location of death, number of emergency room (ER) visits within one month preceding death and administration of chemotherapy in last 14 days of life. Multivariable logistic regression models were used to estimate associations between indicators and oncologist's awareness in PC, intervention of PCT, case discussion at OPMs.

Results: Location of death was home for $58(11 \%)$ patients, ICU or emergency room for 45 (9\%), acute care hospital for 253 (49\%); 185 (36\%) patients visited emergency room within 1 month before death, and 75 (14\%) received chemotherapy within 14 days before death. Only the OPM $(n=179,34 \%)$ independently decreases the odds of receiving chemotherapy in last 14 days of life (OR[CI95\%]:0.5[0.2;0.9]) and the odds of dying at hospital (excluding hospice) $0.3[0.1 ; 0.5])$. Oncologist's awareness in PC $(n=184,35 \%)$ and intervention of the PC team $(n=300,58 \%)$ did not improve independently any indicators. Among patients seen by PCT, median survival after first contact was longer for patients discussed at OPM (75 days) than for those not discussed (29 days). In multivariable analysis, the OPM remains more determinant than survival after first contact to decrease death at hospital.

Conclusion: Multidisciplinary decision-making with referent physician and palliative team is the most critical parameter to improve EOL care. 


\section{INTRODUCTION}

Considerable attention is currently being focused on the impact of integration of palliative care (PC) in oncology [1]. In cancer centres, late referral of patients to specialist PC reflects the persistent barriers to integrated palliative care in oncology [2]. Temel et al. showed that early PC consultation systematically offered to patients diagnosed with advanced non-small cell lung cancer (NSCLC) increased both their survival and quality of life [3]. This study marks a turning point for the integration of PC, pointing out the value of early introduction of the PC team. However, the factors accounting for these results are unclear.

Various models of organisation, intervention and interface of specialist PC with standard care have developed across countries. Efforts to systematically review evidence for effectiveness of PC services lead to ambivalent conclusions, mainly due to insufficient internal and external validity of original studies [4-6]. In their study focusing on "the effectiveness of specialist PC delivered to cancer patients", Higginson et al. state the wide range of services tested, concluding that future studies need to test more specific components of PC team activity [4].

In France, most developed PC services remain hospital based, with inpatient PC centres or hospital PC teams. These teams act as staff counsellors at the patient's bedside in end-of-life situations. They concentrate efforts on the acculturation of the medical and nursing staff being directly in charge of patients. In oncology, this mode of intervention respects the oncologist's role, but its impact on the patient is indirect and highly depends on the degree of organisation and collaboration between both specialties.

In our institution, the PC team has developed a collaborative organisation with the oncology ward in order to reduce persistent barriers and improve the quality of end of life care. This collaboration is based on a weekly onco-palliative meeting (OPM) attended by both the PC team and the oncology staff, who discuss the cases of any advanced cancer patients who could benefit from joint follow-up care. We hypothesized that this OPM, as a key component of the 
PC team activity, may be as important as the on-demand intervention of PC team to improve indicators of quality of end of life care.

Many indicators have been proposed to describe the quality of both outcomes and processes of end-of-life care (i.e. use of health services resources) [7]. In order to estimate the impact of the different modalities of introducing palliative care in the trajectory of cancer patients, we chose process of care indicators. Those developed and evaluated by Earle et al. address three aspects of care [8, 9]: overuse of aggressive treatment resources, unplanned medical encounters, and poor access to PC.

In the present study, we evaluated the impact of oncologist's awareness in PC, clinical intervention of PC team and its timing, multidisciplinary decision-making on the end-of-life care indicators described by Earle et al.[8] 


\section{METHODS}

\section{Design and setting}

Cochin Hospital is a tertiary care hospital treating 6,000 new cancer patients each year, with an oncology department and 3 other medical specialty wards (gastroenterology, pneumology, dermatology) dispensing chemotherapy. We conducted a decedent case series study, using a mortality follow-back approach. Decedent cases were identified by follow-up of all eligible patients recorded in the hospital activity data.

\section{Study population}

All patients diagnosed with solid tumour and having received their last administration of intravenous chemotherapy at Cochin hospital, between June $1^{\text {st }}, 2006$ and December $31^{\text {st }} 2008$ were considered eligible for inclusion in our case series. These patients were identified from the electronic record database (CHIMIO) routinely used to manage patients receiving intravenous chemotherapy.

Patient vital status was identified in three steps. We first cross-matched the CHIMIO database of eligible patients with the hospital discharge data (2007-2008) of 30 acute care hospitals in the Paris area. Then, for patients not recorded to be deceased at discharge, we searched these patients' medical records for a posteriori notification of death. Finally, we completed followup by combining requests to civil administrative services, and telephone interview of the patient's referring primary care physician. All patients deceased during the study period were included.

\section{Organisation of care}

Chemotherapy is prescribed by either the 5 physicians of the oncology ward or the 10 other specialists from other wards qualified in oncology. None of these physicians are involved in palliative care practice, but some of them followed a training program introducing the fundamentals of palliative care, thereby increasing their awareness of palliative care services. 
The patient is then recorded in the CHIMIO database. All 15 prescribers are designated later on as "referent physician". The PC team consists in 2.5 full time equivalent physicians, all PC specialists, 2.5 full time equivalent nurses, 1 secretary assistant, 1 attending psychologist.

There are two different ways of referral to the PC team. One existing with oncology ward, relies on a weekly Onco-Palliative multidisciplinary Meetings (OPM), dedicated to patients deemed incurable and for whom it is necessary to discuss goals of care. The OPM is lead by a physician of the PC team who also records its conclusions in patient's record. It is attended by both the PC team and the oncology staff, i.e. physicians, nurses and head nurses, social workers, psychologists, and secretaries [10]. Patient eligibility for discussion at the OPM is defined by a life expectancy shorter than 6 month, as estimated by the referent physician, who thereby initiates discussion. Discussions take into account expected benefit on survival and quality of life, aggressiveness of care, and patient's preferences. Decisions may be to pursue chemotherapy, associated or not with intervention of the PC team, to propose inclusion in a phase 1 or 2 clinical trial, or to provide PC only. These decisions are then submitted to the patient's wishes. Later on, patients are followed-up jointly by the PC team and the referent physician, and goals of care are updated at following OPMs. Follow-up of patients is made possible, as needed, through outpatient consultations (with the referent physician and/or with the PC team), in day care setting or by admission in acute care setting.

Besides this organized activity supporting an integrated model of care, the PC team also intervenes, upon the request of health professionals from clinical wards, according to their appreciation and without a specific dedicated time, as the OPM, for multidisciplinary decision-making. The intervention addresses symptom management, end-of-life care decisions, coping with a life-threatening illness, illness and prognostic understanding, organisation of referral to hospice, coordination with home care services and networks, or outpatients follow-up. 


\section{Quality indicators}

We evaluated five indicators inspired from those elaborated and validated by Earle et al. [8, 9]: location of death (acute care setting versus home or hospice), number of emergency room (ER) visits within 1 month preceding death (at least one), interval between the last administration of chemotherapy and death (less than 14 days), number of admissions in ICU and number of acute care admissions, within 1 month preceding death. The two last indicators were measured to test their interrelationships with the three first ones.

\section{Data collection of history of care}

Other collected data were age, gender, cancer primary site and three process characteristics: training of referent physician in PC (reflecting his/her actual degree of awareness in PC), whether or not the patient case was discussed at the OPM, intervention or not of the PC team. For patients who had been followed by PC team, we recorded performance status (PS), according to ECOG scale [11], at the time of this first intervention and time interval between first intervention of the team and death

History of care data and quality indicators were collected from computerized clinical databases, completed by search of paper records and interview of referent physicians.

\section{Statistical Analysis}

Location of death (at hospital, excluding hospice versus other location), referral to emergency services ( 1 or more referrals versus none) and the proportion of patients receiving chemotherapy in the last 14 days of life were analysed using logistic regression models. We measured the association of these indicators with history of care (including other indicators), using odds ratio with $95 \%$ Confidence Intervals (95\%CIs). For continuous variables, the linearity of log-odds was checked graphically. The relationships between the quality 
indicators and the colinearity between categorical variables were checked using the Cramer's $\mathrm{V}$ coefficient (a measure of association derived from the Pearson chi-square).

All associations with indicators were studied in both univariable and multivariable analyses. $\mathrm{P}$ value $<0.05$ was considered significant. The goodness-of-fit of the logistic multivariable regression models was tested using the Hosmer-Lemeshow test (small $p$-values indicate a lack of fit of the model). The statistical software SAS (release 9.1; SAS Institute Inc., Cary, NC, USA) was used for all analyses. 


\section{RESULTS}

\section{Study population}

We identified 735 advanced cancer patients who received chemotherapy during the study period (figure 1): 105 (14\%) were not eligible because they were not dead by the end of the pre-defined study period or because they were followed up and cared for in another hospital shortly after diagnosis (i.e. patients choosing to be followed in another cancer centre). For 109 other patients $(14.8 \%)$, whose vital status could not be found in hospital records, the place of birth was either outside France $(n=42)$ or was not informed in hospital admission data $(n=67)$, thus preventing from any request to civil services for vital status. Therefore, we finally analysed 521 patients whose characteristics and history of care are described in table 1 and figure 2 .

Table 1: Patients characteristics and history of care

Total, $\mathrm{N}=521$

\begin{tabular}{lrl}
\hline Mean age at death, years (SD) & 64 & $(13)$ \\
Sex, $\mathrm{n}(\%)$ & & \\
Male & 326 & $(62.6)$ \\
Female & 193 & $(37.0)$ \\
Missing data & 2 & $(0.4)$ \\
& & \\
\hline Primary Cancer site, $\mathrm{n}(\%)$ & & \\
Gastro-intestinal & 110 & $(21)$ \\
Lung & 107 & $(21)$ \\
Urologic & 67 & $(13)$ \\
Melanoma & 67 & $(13)$ \\
Liver and biliary tract & 52 & $(10)$ \\
Other & 118 & $(23)$ \\
\hline
\end{tabular}

Location of death, $\mathrm{n}(\%)$ 
Acute care hospital medicine

Hospice

Home

Emergency room or ICU

$45 \quad(9)$

Other

Missing data

3 (1)

History of care; n (\%)

Referent oncologist trained in palliative care YES

Palliative care team intervention

YES

Missing data

if yes, median survival from inclusion ; days (IQR)

Onco - palliative meeting (OPM)

\section{NO}

341 (66)

Median survival after last chemotherapy, days (IQR)

$55 \quad(25-109)$

Patients receiving chemotherapy in the last 14 days of life, $\mathrm{n}(\%)$

$75(14 \%)$

Emergency room visits, within last 30 days, n (\%)

0

1

$\geq 2$

Missing data

Admissions in ICU, within last 30 days, $\mathrm{n}(\%)$

0

1

$\geq 2$ 
Admissions in acute care setting, within 30 last days, $\mathrm{n}(\%)$

0

If $\geq 1$, median length of stay, days (IQR)

\footnotetext{
* Performance status, as measured by the ECOG [11] http://www.ecog.org/general/perf_stat.html] : 0 : Fully active, able to carry on all pre-disease performance without restriction; 1: Restricted in physically strenuous activity but ambulatory and able to carry out work of a light or sedentary nature, e.g., light house work, office work; 2: Ambulatory and capable of all selfcare but unable to carry out any work activities. Up and about more than $50 \%$ of waking hours; 3 : Capable of only limited selfcare, confined to bed or chair more than $50 \%$ of waking hours; 4: Completely disabled. Cannot carry on any selfcare. Totally confined to bed or chair.
}

\section{Description of indicators}

Location of death was at home for $58(11 \%)$ patients, hospice for $128(25 \%)$, ER or ICU for $45(9 \%)$ and acute care wards for 253 (49\%). Among the 58 patients who died at home, 17 (34\%) died less than 7 days after their last hospital discharge. Among the 128 patients who died in hospice, $14(11 \%)$ died less than 3 days after admission. Among the 253 patients who died in acute care wards, $126(42 \%)$ had been admitted after visiting the ER (unscheduled hospitalisation)

Within their last month of life, 185 (36\%) patients visited ER at least once, 49 (10\%) were admitted to ICU, 242 (46\%) were hospitalized once in an acute care setting and 97 (19\%) twice or more.

The median survival after the last administration of chemotherapy was 55 days (Inter-Quartile Range (IQR):25-109); 75 (14\%) patients received chemotherapy in their last 14 days of life. 


\section{Description of history of care}

Patients were followed by 15 referent physicians. Two of them had followed sensibilisation training in PC and were referent for 101 and 83 patients respectively (35\% overall), whereas 13 doctors with no training in PC each took care of a median number of 16 patients (min-max $1-80)$.

The PC team collaborated to the care for $67 / 101(66 \%)$ and $51 / 83(61 \%)$ patients from the 2 PC qualified physicians versus 182/337 (54\%) patients of the 13 other referent doctors.

Among the 300 patients taken on by the PC team, PS was recorded in 231; 110 (48\%) patients had a PS of 1 or 2 . The median survival after first referral was 51 days. The first contact with the PC team occurred within the last month in a total of $108(40 \%)$. Patients discussed at the OPM (143/300) had a better PS than those not discussed (157/300): PS was $\leq 2$ in 74/143 $(52 \%)$ in patients discussed at the OPM, versus in 36/157 (23\%) out of the OPM $(p<0.001$, Chi-Square test). Discussion at the OPM resulted in earlier entry of the PC team: median survival was 75 days (IQR: 31-182) in discussed patients, versus 29 days (IQR: 6.5-80) in patients not presented at the OPM ( $p<0.001$, Wilcoxon test).

\section{Analysis of location of death}

In univariable analyses (table 2), the odds of dying at hospital (excluding hospice) was significantly decreased by the following variables: PC training of referent oncologist's, intervention of the PC team, discussion of the patient's case at the OPM, survival after the last chemotherapy longer than 60 days. Conversely, and not surprinsingly, this odds was significantly increased with the number of visits to emergency room, and admission to ICU or to acute care hospital. Median survival after the last chemotherapy was 77 days (IQR: 45-142) in patients who died at home or in hospice and 44 (IQR:19-89) for others.

After adjusting for all variables, only the patient's case discussion at the OPM decreases the odds of patients who died at hospital (excluding hospice) by $70 \%$ (adjusted $\mathrm{OR}=0.3$ 
[CI95\%:0.1;0.5],p<0.001). Other indicators remained significantly associated with death at hospital, after adjustment, were ER visits (adjusted OR=2.8 [CI95\%:0.8; 9.8], $\mathrm{p}=0.022$ ) and acute care admissions (global $\mathrm{p}<0.001$; adjusted $\mathrm{OR}=8.3$ [CI95\%:4.2; 16.4] for 1 admission; $\mathrm{OR}=16.2$ [CI95\%:5.9; 44.6] for 2 or more admissions);

In the subgroup of 300 patients followed by the PC team, the time between first intervention of PC team and death and the OPM are both significantly associated with the odds of dying at hospital, whereas the performance status at first evaluation by the PC team was not. In multivariable analysis, only the OPM still decreased this odds by $80 \%$ (adjusted OR=0.2 [CI95\%:0.1;0.5], $\mathrm{p}=0.002)$

\section{Analysis of the use of emergency resources}

No process characteristics significantly decreased the proportion of patients visiting ER at least once in the last 30 days of life, neither in univariable nor in multivariable analyses (table 3). Among other indicators, admission to ICU, and survival after last chemotherapy were not associated significantly with ER visits contrary to acute care admissions which remain significantly associated in multivariable analyses (global p<0.001) (table 3), although patients could also be admitted directly in acute care wards.

Among the 300 patients followed by the PC team, the PS was the only variable associated with the use of emergency services in multivariable analyses: altered PS (3 or 4) doubles the odds of visiting ER at least once (adjusted OR=2.1 [CI95\%:1.0; 4.4], p=0.038). Moreover, survival after the first contact with PC team was not statistically associated with referral to emergency services. These results are consistent with a referral to emergency services for transient, unexpected, acute complication, rather than as the way of managing end-of-life care. 


\section{Analysis of patients receiving chemotherapy in the last 14 days of life}

Median survival after last chemotherapy for patients followed by either PC trained or non trained referent physicians, was 76 and 49 days.

In univariable analyses (table 4), both the intervention of PC team and the discussion of patient's case at the OPM significantly decrease the odds of receiving chemotherapy in the last 14 days of life. In multivariable analyses, the OPM still decreased by $50 \%$ (OR=0.5 [IC 95\%: $0.2-0.9$ ], $\mathrm{p}=0.035$ ) the odds of receiving chemotherapy in the last 14 days of life.

Among the 300 patients followed by the PC team, the OPM was the only factor reducing the odds of receiving chemotherapy in the last 14 days of life by $60 \%$, in univariable analyses, but this effect did not reach statistical significance in multivariable analysis (adjusted OR=0.4 [IC 95\%: $0.1-1.2] \mathrm{p}=0.089)$. 
Table 2: Location of death, univariable and multivariable logistic regression analyses.

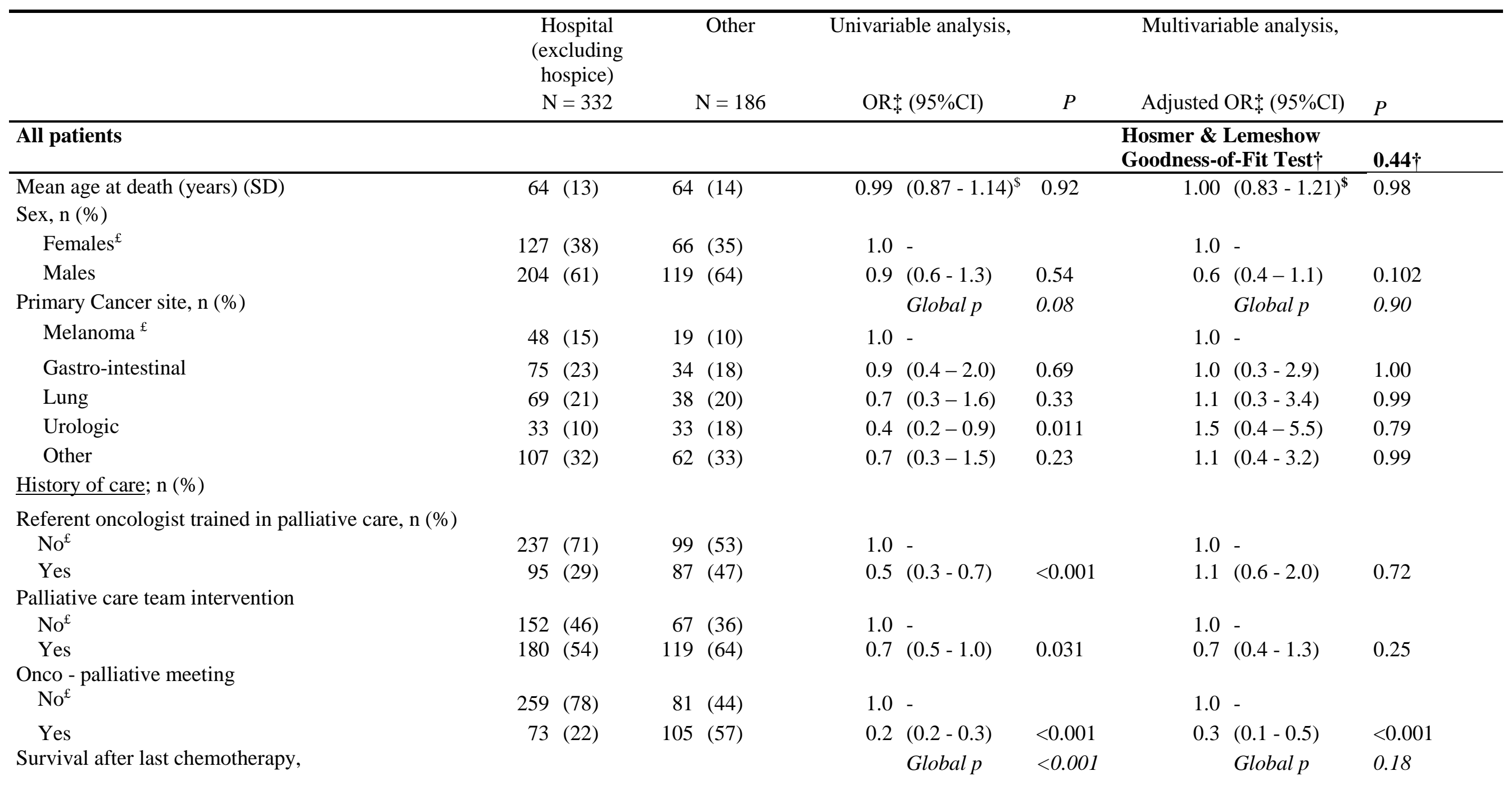




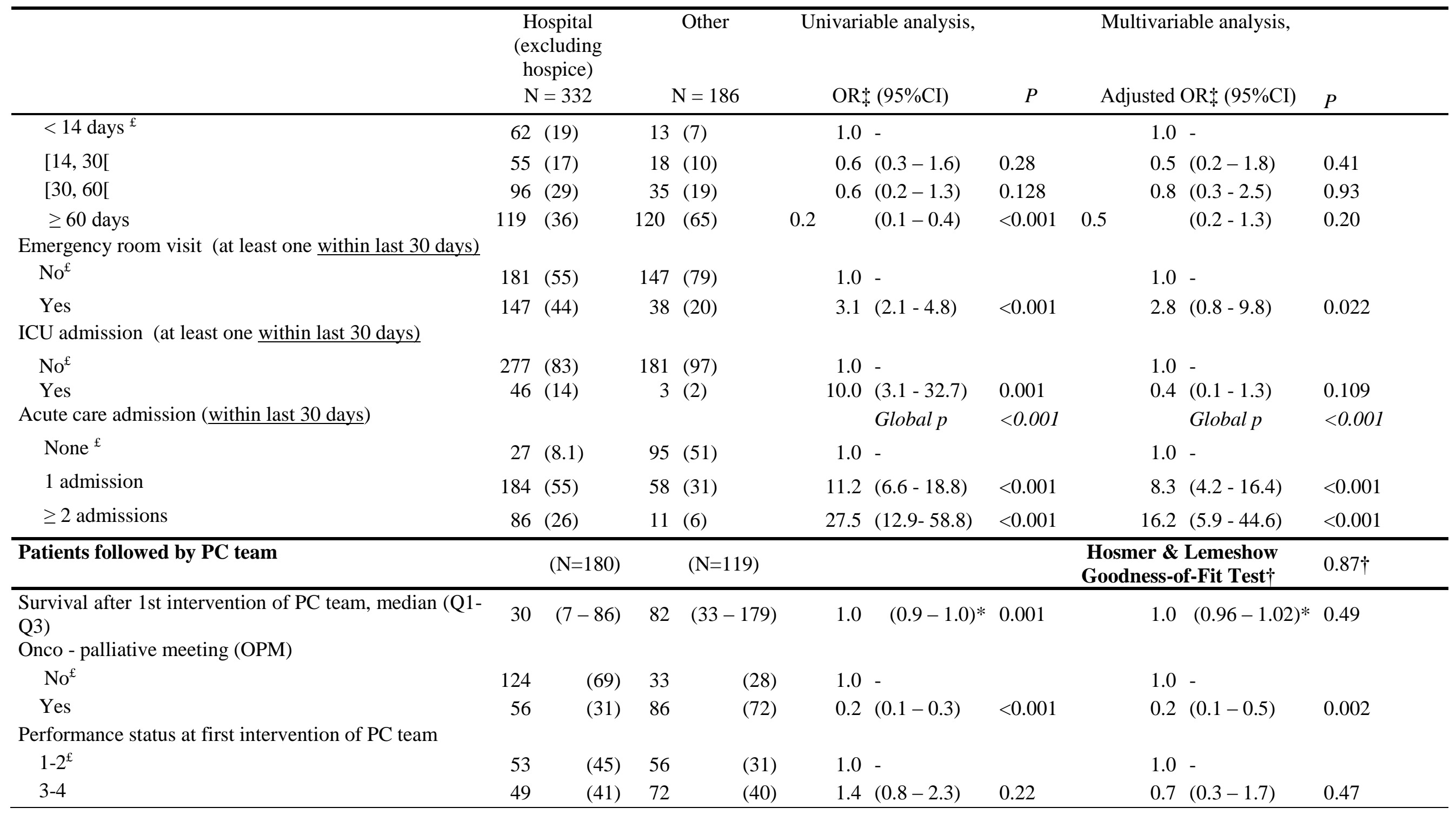


† Odds Ratio for death at Hospital (excluding hospice) versus Other. For multiple comparisons testing difference with the reference category (primary cancer site, survival after last chemotherapy, acute care admission), p-values were adjusted using Dunnett correction. For the subgroup of patients followed by PC team, the multivariable logistic regression models included age, gender, primary cancer site, and training of referent physician in PC.

$\uparrow$ The null hypothesis cannot be rejected, indicating that the model fits the data (small p-values indicate a lack of fit of the model).

$\$$ for 10 years increase of age.

${ }^{£}$ Reference category for univariable and multivariable logistic regression. For cancer site, we chose the cancer site with the lowest risk of dying at home or hospice as reference category in order to facilitate interpretation (odds ratio greater than one). In univariable analysis, this category was "melanoma" and we used it as reference category for all analyses.

* for 14 days increase of survival. 
Table 3: Use of emergency services within last 30 days, univariable and multivariable logistic regression analyses.

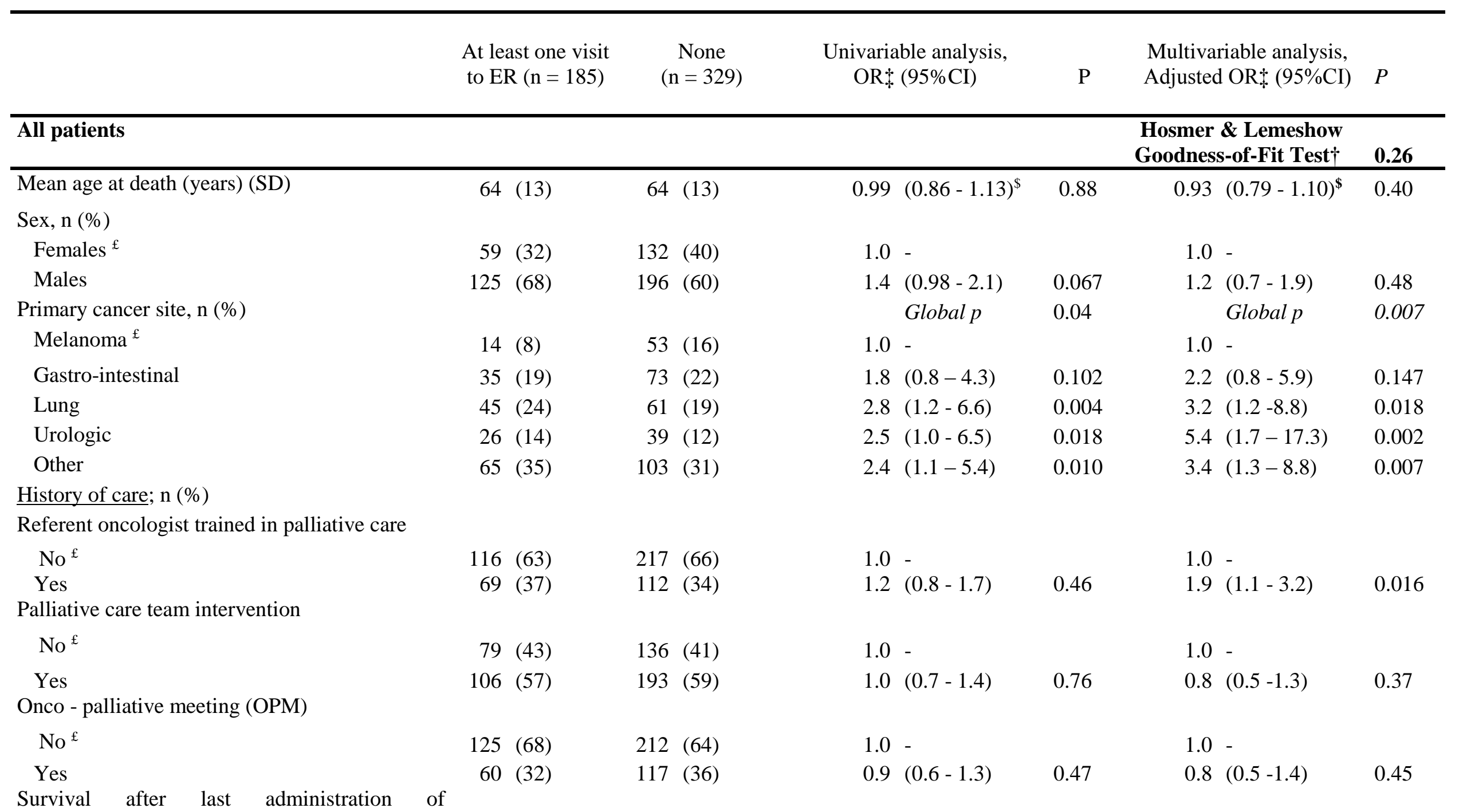




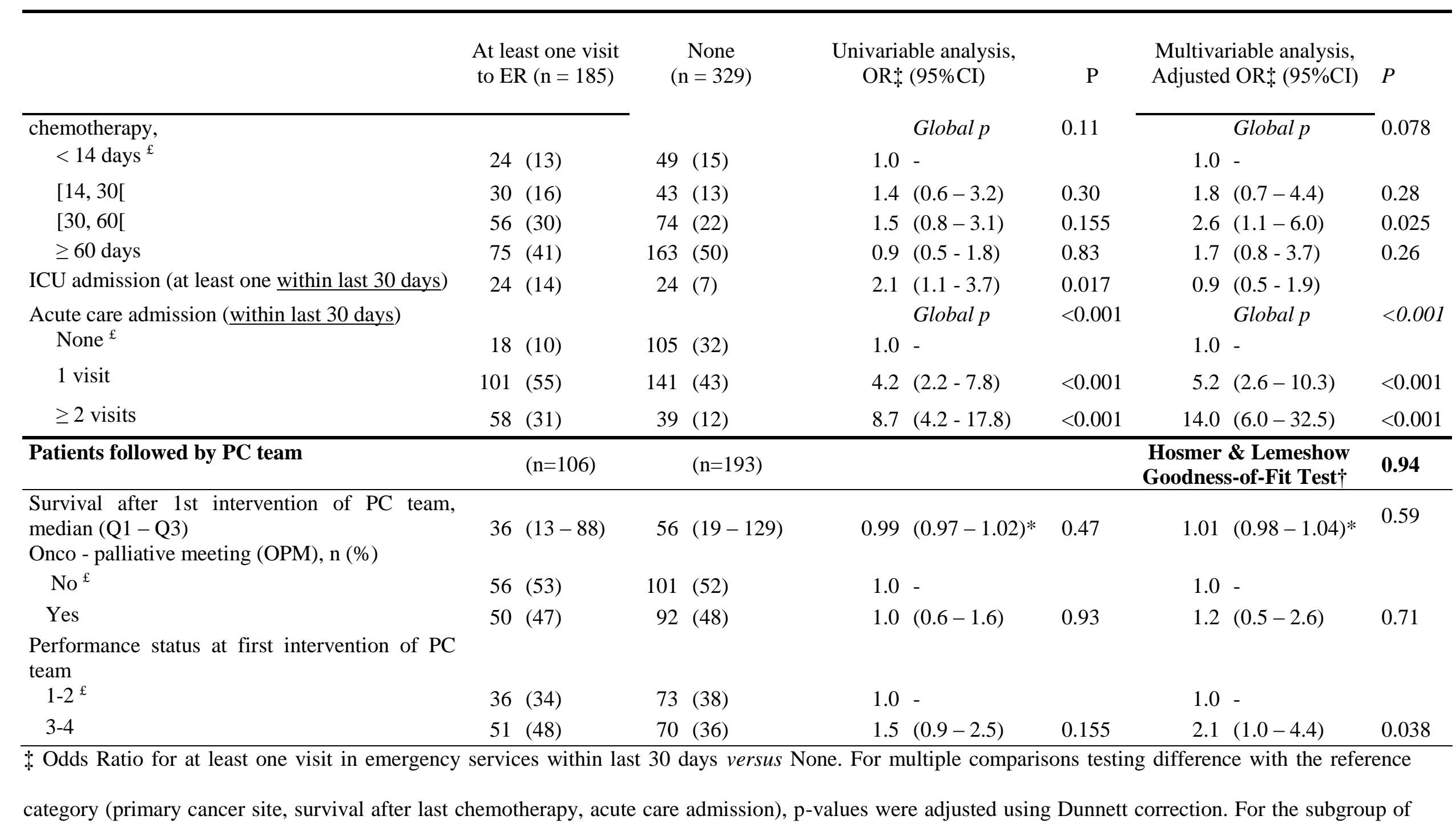


patients followed by PC team, the multivariable logistic regression models included age, gender, primary cancer site, and training of referent physician in PC.

$\uparrow$ The null hypothesis cannot be rejected, indicating that the model fits the data (small p-values indicate a lack of fit of the model).

$\$$ for 10 years increase of age.

${ }^{£}$ Reference category for univariable and multivariable logistic regression. For cancer site, we chose the cancer site with the lowest risk of dying at home or hospice as reference category in order to facilitate interpretation (odds ratio greater than one). In univariable analysis, this category was "melanoma" and we used it as reference category for all analyses.

* for 14 days increase of survival 
Table 4: Patients receiving chemotherapy in the last 14 days of life, univariable and multivariable logistic regression analyses

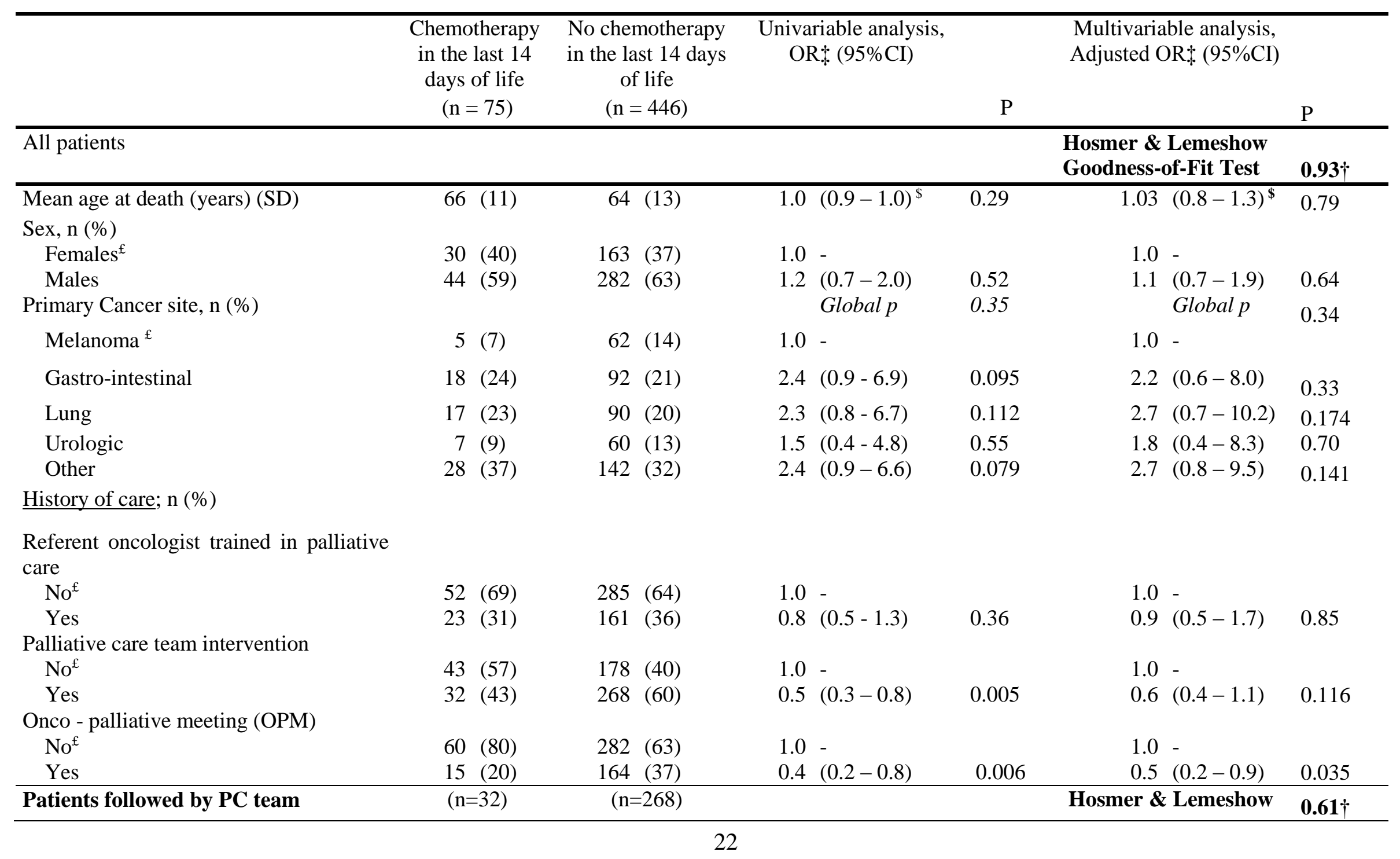




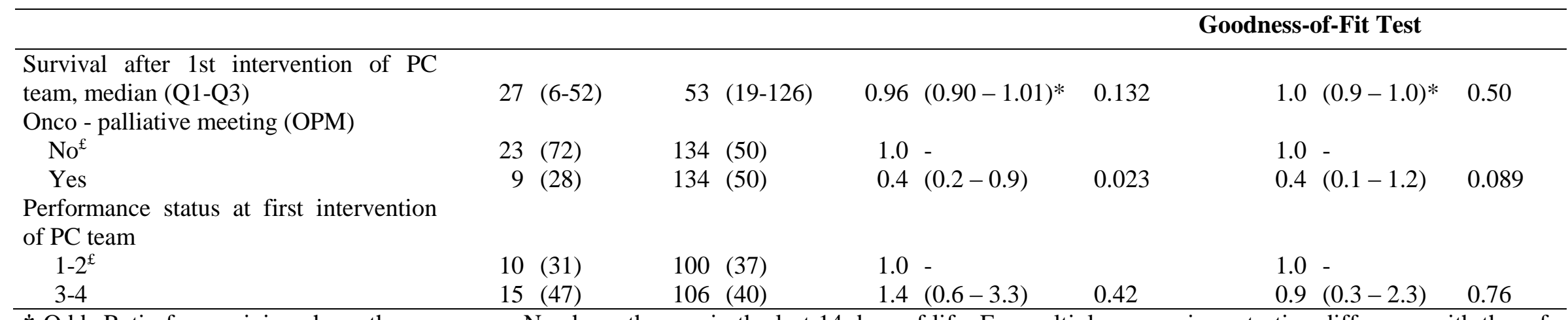

† Odds Ratio for receiving chemotherapy versus No chemotherapy in the last 14 days of life. For multiple comparisons testing difference with the reference

category (primary cancer site, survival after last chemotherapy, acute care admission), p-values were adjusted using Dunnett correction. For the subgroup of patients followed by PC team, the multivariable logistic regression models included age, gender, primary cancer site, and training of referent physician in PC.

$\uparrow$ The null hypothesis cannot be rejected, indicating that the model fits the data (small p-values indicate a lack of fit of the model).

$\$$ for 10 years increase of age.

${ }^{\mathfrak{f}}$ Reference category for univariable and multivariable logistic regression. For cancer site, we chose the cancer site with the lowest risk of dying at home or hospice as reference category in order to facilitate interpretation (odds ratio greater than one). In univariable analysis, this category was "melanoma" and we used it as reference category for all analyses.

* for 14 days increase of survival. 


\section{DISCUSSION}

In this study we retrospectively analyzed a case series of 521 deceased cancer patients to evaluate the impact of oncologist's awareness in palliative care (PC), timing of the PC team intervention, and multidisciplinary decision-making on process quality indicators of end of life care. We found that the active collaboration between referent physicians and PC team, through weekly OPMs was the unique independent factor decreasing the odds of dying at hospital (excluding hospice) by $70 \%$ and decreasing the odds of receiving chemotherapy in last 14 days of life by $60 \%$. These effect sizes are of clinical importance for both palliative care specialists and oncologists. The clinical intervention of PC team was not significantly associated with any indicators. However, in the subgroup of patients who were seen by the PC team, patients discussed in OPM were seen earlier by the PC team (better performance status at first contact and longer follow up before death). Knowing that the PC team can be sollicitated by two different ways (through the OPM or on demand) our data show that the first mode of referral favors timely intervention of PC team. To our knowledge, no such data has been published in the context of a European cancer care health organisation.

While evaluating the performance of a given structure of care, it is necessary to account for all decedents among patients who have been cared for in this structure. The choice of a follow-back of decedents approach does not account for all patients prospectively expected to die, but rather for all patients deceased during a given period of time [12-14]. Interestingly, retrospective and prospective measures of Earle's indicators identified similar patterns of endof-life care [15]. However in our study, for $14.8 \%$ patients, whose vital status could not be found in hospital records, incomplete administrative data made it impossible to request civil services for vital status. Some of these patients could have died during the study period. It is difficult to make any assumptions concerning their location of death. Home palliative care services are not highly developed in France and have to be sollicitated by hospital-based PC 
teams, at discharge of patient. Patients who have not been referred to and followed by any PC team during their hospital stay are therefore unlikely to be referred to any home care services. Conversely, when lost to follow up by hospital teams, they can die at home or in another acute care hospital as well. The underestimation of the proportion of patients who died at home, in remaining data should therefore be slight, if it exists.

Compared to the standards proposed by Earle et al [9] (less than $17 \%$ of deaths in acute care hospital, less than $10 \%$ of patients still treated within 14 days before death, and less than $4 \%$ of patients having visited ER more than once within last month of life), our data showed respectively $58 \%, 14 \%$ and $5 \%$ for the three indicators. Concerning the location of death, preference to die at home is often reported as a goal of care [16]. However, the congruence of preferred and actual place of death is rarely good [17]. The Dying Well in Europe study [18] showed the high variability of rates of death at home in cancer patients across European countries (from $12 \%$ to $45 \%$ ).

Although the components of the early PC consultation have been described in more detail, as well as the patient's factors associated with its duration [19][20], the respective roles of the earliness of consultation and of the actual sharing of decision-making between PC specialists and oncologists remained unexplored. As stated by ASCO back in 1998, it is the oncologist's responsibility "to care for their patients in a continuum that extends from the moment of diagnosis throughout the course of illness" [21]. Systematic early PC consultation may make it more difficult for them not to withdraw (or be withdrawn) from care relationship at the end of life. Despite recent progress [22], oncologists still refer their patients to the PC team late in the trajectory of illness [2]. The role of the oncologist is known to be crucial for integrating PC in oncology practice [23]. Kao et al. [24] found that the oncologist was the only predictor for continuing chemotherapy in the last 4 weeks of life. In our organisation, the OPM allows to systematically discuss goals of care and to anticipate the difficult period of transition of 
care. The continuous collaboration of the PC team with oncologists facilitates its early intervention. Temel et al. [3] demonstrated in a randomized control trial the effectiveness of early and longitudinal participation of the PC team in newly diagnosed metastatic NSCLC is decreasing aggressive care, lowering the rate of depression and increasing quality of life and survival. We assume that, in the setting of this trial, oncologists adhered to the concept of early PC.

Improving the quality of outcome in end-of-life patients requires clinical research in two different directions: the first is to improve the ability of the physicians to evaluate the prognosis and life-expectancy of advanced cancer patients. Several clinical and biological parameters have been shown to correlate with very poor survival and several simple scores have been described [25-27] and are under prospective evaluation. The second direction is to define and evaluate the organisation and procedures the most susceptible to impact quality of care and patient outcomes. Rigorous evaluation requires both actual implementation of these procedures and use of validated indicators to account for quality of care. This paper participates to this research providing with the first measure of such indicators in a French centre and showing the critical role of the OPM. To initiate OPMs appears more important than the PC training of the referent physician (although it helps to introduce the model), and more important than the timing of entry of the PC team. Indeed, early PC is much easier and more effective, if preceded by a shared decision on the treatment strategy. A multicentric study is underway in the Paris area to collect data, from various cancer care centres where the organisation of PC highly differs from one another. This study should provide more results to evaluate both the pertinence and external validity of Earle's indicators and to evaluate the impact of different organisations of care.

In conclusion, our results indicate that a weekly meeting with PC team and oncology staff offering a multidisciplinary decision-making for non-curable patients is critical factor to 
improve quality indicators. This suggests that beyond the earliness of clinical intervention of PC team, the quality of collaboration and the structuring of discussion may be a necessary condition to integrate palliative care in oncology. Early "seed" of the PC team is necessary but not sufficient. Support by the oncologic "soil" is needed and lead to integrative medicine.

\section{ACKNOWLEDGEMENTS}

We thank Maryvonne Guillard, Nathalie Moreau, Françoise Travers (nurses of the PC team), Hélène de la Ménardière (psychologist) and all referent physicians, and health professionals who participated to the onco-palliative meeting and thereby contributed to generate the data.

\section{FUNDING}

Foundation Martine Midy supported this research through a grant to Vincent Montheil (VM).

\section{Conflict of interest: None}




\section{REFERENCES}

1. Bruera E, Hui D. Integrating supportive and palliative care in the trajectory of cancer: establishing goals and models of care. J Clin Oncol 2010; 28(25):4013-7.

2. Hui D, Elsayem A, De la Cruz M, et al. Availability and integration of palliative care at US cancer centers. JAMA 2010; 303(11):1054-61.

3. Temel JS, Greer E, Gallagher S. Early palliative care for patients with metastatic NonSmall-Cell lung cancer. N Engl J Med 2010; 363(8):733-741.

4. Higginson IJ, Evans CJ. What is the evidence that palliative care teams improve outcomes for cancer patients and their families? Cancer J 2010; 16(5):423-35.

5. Lorenz KA, Lynn J, Dy SM, et al. Evidence for improving palliative care at the end of life: a systematic review. Ann Intern Med 2008; 148(2):147-59.

6. Zimmermann C, Riechelmann R, Krzyzanowska M, Rodin G, Tannock I. Effectiveness of specialized palliative care: a systematic review. JAMA 2008; 299(14):1698-709.

7. Pasman HR, Brandt HE, Deliens L, Francke AL. Quality indicators for palliative care: a systematic review. J Pain Symptom Manage 2009; 38(1):145-156.

8. Earle CC, Park ER, Lai B, Weeks JC, Ayanian JZ, Block S. Identifying potential indicators of the quality of end-of-life cancer care from administrative data. J Clin Oncol 2003; 21(6):1133-8.

9. Earle CC, Neville BA, Landrum MB, et al. Evaluating claims-based indicators of the intensity of end-of-life cancer care. Int J Qual Health Care 2005; 17(6):505-9.

10. Durand JP, Goldwasser F. [New challenges in medical oncology. From medical oncology to oncological medicine]. Ann Med Interne (Paris) 2003; 154(3):131-2.

11. Oken MM, Creech RH, Tormey DC, et al. Toxicity and response criteria of the Eastern Cooperative Oncology Group. Am J Clin Oncol 1982; 5(6):649-55. 
12. Bach PB, Schrag D, Begg CB. Resurrecting treatment histories of dead patients: a study design that should be laid to rest. JAMA 2004; 292(22):2765-70.

13. Barnato AE, Farrell MH, Chang CC, Lave JR, Roberts MS, Angus DC. Development and validation of hospital "end-of-life" treatment intensity measures. Med Care 2009; 47(10):1098-105.

14. Earle CC, Ayanian JZ. Looking back from death: the value of retrospective studies of end-of-life care. J Clin Oncol 2006; 24(6):838-40.

15. Setoguchi S, Earle CC, Glynn R, et al. Comparison of prospective and retrospective indicators of the quality of end-of-life cancer care. J Clin Oncol 2008; 26(35):5671-8.

16. Kaldjian LC, Curtis AE, Shinkunas LA, Cannon KT. Goals of care toward the end of life: a structured literature review. Am J Hosp Med 2009; 25:501-511.

17. Bell CL, Somogyi-Zalud E, Masaki KH. Methodological review: measured and reported congruence between preferred and actual place of death. Palliat Med 2009; 23(6):482-90.

18. Cohen J, Houttekier D, Onwuteaka-Philipsen B, et al. Which patients with cancer die at home? A study of six European countries using death certificate data. J Clin Oncol 2010; 28(13):2267-73.

19. Temel JS, Jackson VA, Billings JA, et al. Phase II study: integrated palliative care in newly diagnosed advanced non-small-cell lung cancer patients. J Clin Oncol 2007; 25(17):2377-82.

20. Jacobsen J, Jackson V, Dahlin C, et al. Components of early outpatient palliative care consultation in patients with metastatic nonsmall cell lung cancer. J Palliat Med 2011; 14(4):459-64.

21. Cancer care during the last phase of life. Adopted on 20 February 1998 by the American Society of Clinical Oncology J Clin Oncol 1998, 16 1986-96. 
22. Osta BE, Palmer JL, Paraskevopoulos T, et al. Interval between first palliative care consult and death in patients diagnosed with advanced cancer at a comprehensive cancer center. J Palliat Med 2008; 11(1):51-7.

23. Maltoni M, Amadori D. Palliative medicine and medical oncology. Ann Oncol 2001; 12(4):443-50

24. Kao S, Shafiq J, Vardy J, Adams D. Use of chemotherapy at end of life in oncology patients. Ann Oncol 2009; 20(9):1555-9.

25. Durand JP, Mir O, Coriat R, Cessot A, Pourchet S, Goldwasser F. Validation of the Cochin Risk Index Score (CRIS) for life expectancy prediction in terminally ill cancer patients Support Care Cancer 2011:Apr 16. [Epub ahead of print].

26. Maltoni M, Nanni O, Pirovano M, et al. Successful validation of the palliative prognostic score in terminally ill cancer patients. Italian Multicenter Study Group on Palliative Care. J Pain Symptom Manage 1999; 17(4):240-7.

27. Morita T, Tsunoda J, Inoue S, Chihara S. The Palliative Prognostic Index: a scoring system for survival prediction of terminally ill cancer patients. Support Care Cancer 1999; 7(3):128-33. 


\section{Figure and Tables}

Figure 1 : Flow diagram for selection of decedents

Figure 2: Flow chart of organisation of care

* 16/64 of these patients discussed at the OPM, were actually followed by specialist referent physicians who did not participate to the OPM. These patients happened to be referred to oncology day care unit for chemotherapy administration and their case were brought up at the OPM by residents in charge of this unit. 